ISSN electrónico: 2602-8069

\title{
ESTUDIO PRELIMINAR DE SITIO Y COMPETITIVIDAD EN PROPUESTAS TURÍSTICAS COMUNITARIAS: ZULETA PAISAJE CULTURAL
}

Preliminary study of site and competitiveness in community tourist proposals: Zuleta cultural Landscape

\author{
Diana Enríquez P. \\ Universidad Central del Ecuador \\ deenriquez@uce.edu.ec \\ Paola Flores $\mathrm{P}$. \\ Universidad Tecnológica Equinoccial \\ fpjp97202@ute.edu.ec \\ Andrea Vásquez $\mathrm{O}$. \\ Universidad Tecnológica Equinoccial \\ voap9721@ute.edu.ec
}




\section{Diana Enríquez - Paola Flores - Andrea Vásquez \\ Estudio preliminar de sitio y competitividad en propuestas turísticas comunitarias: Zuleta paisaje cultural}

\section{RESUMEN}

La importancia del presente estudio radica en brindar a la comunidad de Zuleta la posibilidad de ejecutar una actividad económica alternativa, que permita el uso adecuado de los recursos naturales y culturales, a fin de conservar el patrimonio y dinamizar la economía local. En Zuleta, la principal actividad económica es la agropecuaria, sin embargo, existe la posibilidad de gestionar el turismo. Por tanto, el objetivo de la presente investigación es determinar el tipo de producto turístico que resulta ser Zuleta, el método utilizado es cuantitativo-cualitativo, y el instrumento es la ficha técnica de evaluación preliminar de sitio propuesta por The Nature Conservancy. Es un marco de planificación que considera los sistemas ecológicos y la necesidad de inclusión de las comunidades. Los resultados muestran que Zuleta puede ser considerado como producto turístico tipo A. Una vez obtenidos estos resultados, se aplica una segunda metodología cuyo objetivo es desarrollar un diagnóstico del producto turístico que actualmente ofrece Zuleta, y su competitividad en el mercado. Se basa en un análisis de momentos de verdad fundamentado por la metodología del Triángulo del Servicio propuesto por Karl Albrecht en 1988, con el fin de identificar características positivas y negativas que permitan la potenciación y corrección de dicho producto. Los resultados muestran que Zuleta cuenta con elementos esenciales como accesibilidad y atractivos turísticos; sin embargo, la poca predisposición de la comunidad y la limitada adecuación técnica representan debilidades en cuanto a calidad. Además, se establece una comparación en la que el beneficio esperado por el cliente es superior al beneficio percibido.

Palabras clave: Conservación de sitios, Paisaje Cultural, Competitividad.

\section{ABSTRACT}

The importance of this study lies in providing the community of Zuleta with the possibility of executing an alternative economic activity that allows the proper use of natural and cultural resources, in order to conserve the heritage and boost the local economy. In Zuleta the main economic activity is agriculture, but there is the possibility of managing tourism. Therefore the objective of this research is to determine the type of tourism product that turns out to be Zuleta, the method used is qualitative and quantitative and the instrument is the evaluation form proposed by The Nature Conservancy; it involves a planning framework that contemplates the ecological systems and the necessity to make communities more inclusive. The results showed that Zuleta can be considered as a tourist product type A, once obtained these results it follows the application of the second methodology which involves a diagnostic of the tourist product that Zuleta offers nowadays and its competitiveness in the market, it consists of an analysis of truth moments based on the methodology known as The Service Triangle proposed by Karl Albrecht in 1988 in order to identify positive and negative characteristics, that allow the strengthening and correction of the product, the results identified that it has essential elements such as accessibility and tourist attractions. Nevertheless, the few community predisposition and the limited technic adaptation represent weaknesses in quality; also it is set a comparison in which the profit the client wants is superior to the profit perceived.

Key words: Conservation of sites, Cultural Landscape, Competitiveness. 


\section{INTRODUCCIÓN}

Zuleta es una comunidad que pertenece a la provincia de Imbabura, y corresponde al municipio de Ibarra, se caracteriza por ser emprendedora integrada por familias indígenas que mantienen costumbres y tradiciones propias de su pueblo; además, cuenta con un paraje natural y cultural inconfundible. En la comunidad se llevan a cabo varias actividades, en el sector económico de servicios, la principal actividad que se realiza es el comercio al por mayor y por menor con el $20.54 \%$ de la totalidad; la segunda actividad en esta rama es el alojamiento y los servicios de alimentos y bebidas lo que representa el 3.98\%de la totalidad (INEC, 2010).

Por esta razón, se ha pensado en varias iniciativas que permitan conservar el patrimonio natural y cultural de la región. Según UNESCO (2017), el patrimonio natural engloba las formaciones físicas y biológicas de valor excepcional como el bosque protector denominado "Zuleta y Anexos Cía. Ltda." Que, según lo estipulado en el PDOT de la parroquia Angochagua (Gobierno Autónomo Desentralizado de la Parroquia Angochagua, 2015), fue declarado por el Ministerio del Ambiente mediante resolución N. 022 del 1 de junio de 1995 con alta prioridad de conservación y se encuentra localizado en las parroquias de Mariano Acosta en el cantón Pimampiro, y las parroquias de La Esperanza y Angochagua del cantón Ibarra. En cuanto al patrimonio cultural inmaterial, Zuleta cuenta con técnicas artesanales tradicionales reconocidas como el bordado, considerado una industria de manufactura según el Plan de Desarrollo y Ordenamiento Territorial de Ibarra, además del trabajo en madera y la gastronomía.

En definitiva, Zuleta cuenta con patrimonio natural y cultural que le permite generar una propuesta de desarrollo integral fundamentado en la herramienta de paisaje cultural desarrollado por UNESCO en 1992, que considera la puesta en valor de un territorio basado en su patrimonio con elementos como sociedad, ambiente, construcción y productividad. Dentro de la unidad de productividad, se establece la posibilidad de gestionar el turismo en la zona a través de la puesta en valor del patrimonio a fin de mejorar la calidad de vida en la población (Gobierno Autónomo Descentralizado de San Miguel de Ibarra, 2012).Cabe destacar que el presente trabajo investigativo aborda la posibilidad de gestionar la actividad turística en el territorio y determina si existen las condiciones necesarias, por lo que se deja abierta la posibilidad de nuevos estudios en cuanto al patrimonio cultural y natural de la localidad.

\section{METODOLOGÍA}

Para obtener la información presentada en este documento, se llevaron a cabo dos metodologías. La primera que se aplica es la determinada por The Nature Conservancy, en la que, mediante el instrumento de ficha técnica de evaluación preliminar de sitio fundamentada en el "Proceso para la planificación y conservación de sitios" (PCS), se identifican las condiciones turísticas de Zuleta a fin de establecer qué tipo de producto turístico resulta ser y sus posibilidades de potenciación.

EI PCS es una herramienta metodológica que considera los sistemas ecológicos y la necesidad de inclusión de grupos sociales, cuyo centro de aplicación define el paisaje de conservación tanto para especies como para comunidades. Inicialmente, la planificación de la conservación de las áreas se concentra en la identificación de sitios prioritarios, y en el diseño y manejo de áreas naturales protegidas (March, Cabral y Echeverría, 2010). Actualmente, se ha ampliado su enfoque de áreas naturales protegidas y zonas de amortiguamiento a la protección de la biota y los procesos que la sostiene a nivel paisaje (The Nature Conservancy, 1999).

De acuerdo con Kiesecker, Comendant, Grandmason y Elizabeth (2007), el proceso de la Planificación para la Conservación de Sitios (PCS) ha resultado ser bastante útil, flexible y adaptable a diferentes escalas y sitios con diferentes contextos naturales y culturales. Una de las grandes ventajas de la PCS es que, de acuerdo al nivel de planificación y manejo que se encuentre un sitio determinado, no es necesario aplicar todos los pasos del proceso, solo se toma la herramienta metodológica que se necesita. 
Diana Enríquez - Paola Flores - Andrea Vásquez

Estudio preliminar de sitio y competitividad en propuestas turísticas comunitarias: Zuleta paisaje cultural

Evaluación de factibilidad

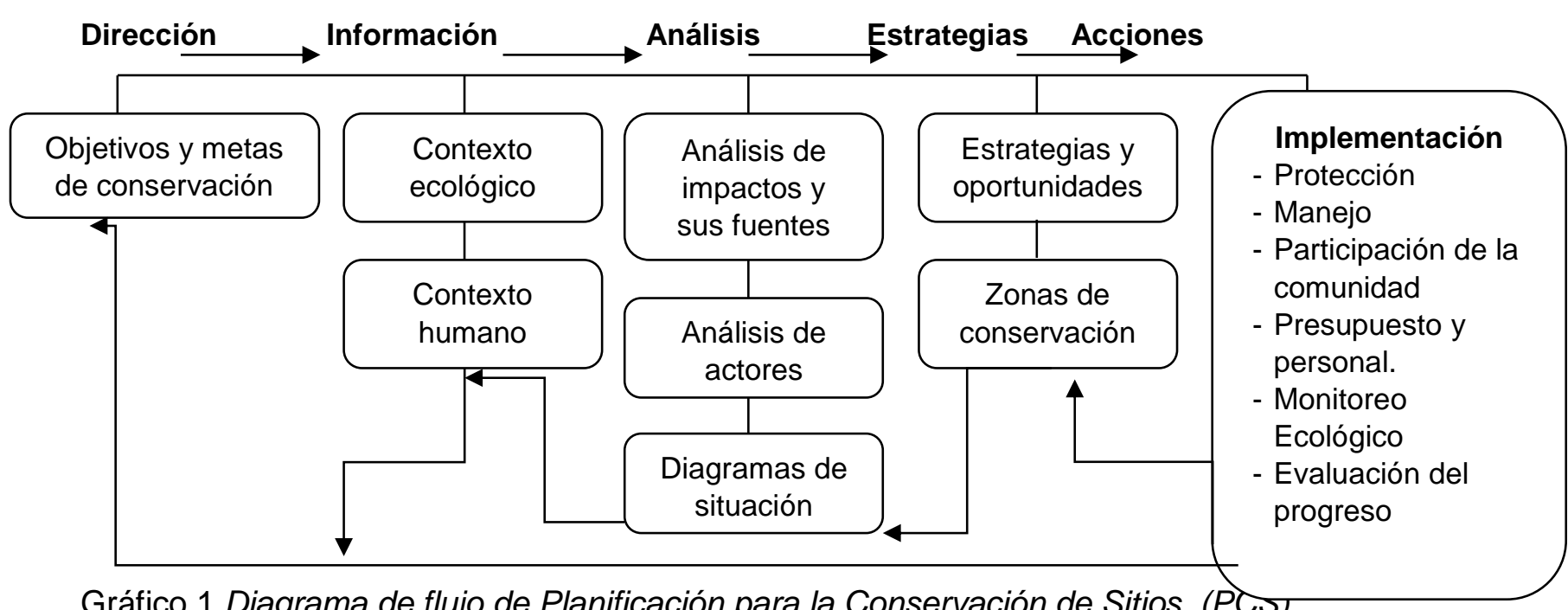

Fuente:The Nature Conservancy (1999)

Elaborado por: The Nature Conservancy

\section{Retroalimentación por medidas de éxito}

Por otro lado, el instrumento usado es la ficha técnica de evaluación preliminar de sitio que analiza diez puntos estratégicos para considerar a un lugar adecuado para ejercer la actividad turística; estos elementos son el recurso turístico, la accesibilidad, la protección en cuanto a posibles riesgos, seguridad, manejo administrativo, financiamiento, compromiso de actores, aporte a la comunidad, infraestructura y facilidades, alcance y mercado potencial, cabe mencionar que no es la única herramienta que existe para este propósito.

Existen varias formas de valoración turística como Big Data en el cual se evalúa servicios o atractivos turísticos con valoraciones positivas, negativas y neutras (Manzato y Rejowski, 2007) o apreciaciones en función de una escala de Likert con valoraciones de muy adecuado, adecuado, poco adecuado o no adecuado (Amaya Molinar y Magaña Espinoza, 2017). En este caso, se aplican los parámetros TNC, debido a que se considera a la comunidad como parte integral de la actividad turística, las otras modalidades suelen valorar infraestructura, servicios y atractivos sin considerar a la comunidad que es el eje promotor del desarrollo turístico, y sobre quienes pueden recaer efectos propios de la actividad turística, de los cuales existen dos visiones: el positivismo y negativismo.

En la visión positiva u optimista, según Pereiro (2013), varios autores destacan el intercambio de cultura, los incentivos a la producción artesanal y más que nada los réditos económicos. Para Alvear, Quishpe y Enríquez (2016), la práctica del turismo comunitario es una estrategia se desarrolla impulsada desde los gobiernos. En cuanto al negativismo, Pereiro (2013) establece varios efectos como conflictos intrafamiliares, transformación de los rituales, deterioro de los recursos naturales, entre otros, lo que pone en una balanza la decisión de ejecutar o no la actividad turística en comunidades. En una buena planificación del turismo comunitario, el eje principal para la gestión e implementación del mismo debe ser la comunidad local (Alvear, Quishpe y Enríquez, 2016); en este caso en particular se hace notar la importancia de utilizar herramientas de medición que consideren a la comunidad.

En Zuleta, se realiza una valoración a través de los parámetros TNC para especificar cuantitativamente la categorización del producto turístico, con el fin de determinar el mercado meta al cual debe dirigirse la propuesta única de valor y la planificación estratégica. 


\section{Estudio preliminar de sitio y competitividad en propuestas turísticas comunitarias: Zuleta paisaje cultural}

La segunda metodología engloba un diagnóstico del producto turístico que actualmente se ofrece para determinar qué tan competitivo es este producto en el mercado. Esta metodología trata de un análisis de momentos de verdad fundamentado por la metodología del Triángulo del Servicio propuesto por Karl Albrecht en 1988, con el fin de identificar características positivas y negativas que permitan la potenciación y corrección de dicho producto en cuanto al servicio que se ofrece a los actuales visitantes.

Teniendo en cuenta que el producto turístico, según Miguel Ángel Acerenza, es un conjunto de prestaciones materiales e inmateriales que se ofrecen con el propósito de satisfacer deseos o expectativas de un turista, en función de los componentes básicos que lo integran: atractivos, facilidades y acceso (Colina, 2015). Resulta de vital importancia analizar las condiciones de la comunidad para determinar su competitividad, ya que es claro que un producto, servicio o precio pueden ser fácilmente imitables.

\section{Según Carlzon (1987, p. 18), un momento de verdad es:}

Cuando los momentos de verdad no se manejan bien, la calidad del servicio regresa a la mediocridad. La suma de las evaluaciones colectivas de todos los clientes, establece en su mentalidad la imagen de la organización en términos de calidad del servicio.

Por otro lado, el Triángulo del Servicio es un modelo que considera los siguientes elementos clave: la estrategia de servicio, el personal y los sistemas de servicio. Los tres deben estar enfocados en el cliente como elemento central de la gestión (Albrecht y Zemke, 1988).



Gráfico 2 El triángulo del servicio

Fuente: Albrecht y Zemke (1988)

Elaborado por: Karl Albrecht

El triángulo representa un proceso en el cual el centro de aplicación es el cliente. El primer paso es identificar a quién vamos a servir o atender, y así entender sus necesidades y motivaciones. El segundo paso es definir la estrategia de servicio, ya que la oferta deberá servir para diferenciarse de la competencia; para esto, se debe comprender que son los clientes los que deben percibir a la organización de manera positiva. El tercer paso es definir 


\section{Estudio preliminar de sitio y competitividad en propuestas turísticas comunitarias: Zuleta paisaje cultural}

el sistema de servicio, el cual incluye procedimientos y normas. El cuarto paso es el personal que representa un factor fundamental debido a que ayudará a diferenciarse y lograr impactar positivamente en los clientes. Es por esta razón que se considera a las personas como un elemento con peso propio en el modelo. Cada uno de estos cuatro elementos debe colaborar con los demás. Eso es lo que representan cada una de las seis líneas del triángulo (García, 2014).

De acuerdo con Requena y Serrano (2007), la calidad del servicio es la amplitud de la diferencia que existe entre las expectativas de los clientes y sus percepciones en función de la tangibilidad, confiabilidad, capacidad de respuesta y empatía.Es por eso que las autoras afirman que convertise en un ejecutor sobresaliente de servicios debe ser la meta de un camino de procesos de alta calidad.

Para Prieto (2014), la cadena de valor cuenta con momentos en el que el cliente puede advertir experiencia positiva o experiencias negativas. Los autores investigados coinciden en que el análisis de los momentos de la verdad contribuye a la generación de calidad en el servicio y permiten la competitividad en el mercado. Según Porter (1982), el liderazgo en costos, la segmentación del mercado y la diferenciación del producto son estrategias para la generación de ventajas competitivas. La diferenciación del producto es la estrategia que se pretendería implementar depués de realizar el análisis de momentos de verdad, ya que la mejor diferenciación es la realizada a través de las caraterísticas del servicio.

Por esta razón, en el desarrollo del presente documento se mide la percepción de los visitantes mediante la metodología antes mencionada y el uso del instrumeto encuesta para establecer estrategias que coadyuven a la competitividad del destino Zuleta, basadas en tres macroelementos de análisis del servicio que identificarán las falencias en cuanto a calidad.

Entre los macroelementos considerados tenemos: cobertura de servicios básicos para los visitantes, atractivos turísticos de lugar y organización comunitaria. En primer lugar, se establece las condiciones básicas para la prestación de un servicio como el acceso al sitio, servicios básicos y las posibilidades de tener a disposición lugares para pernoctar y comer. En segundo lugar, se considera la existencia de senderos en el bosque; la calidad de las artesanías y bordados; actividades de recreación que eviten que este sea un lugar de paso, En tercer lugar, se considera que la comunidad ofrece aseo y asepsia en los servicios brindados, da a conocer políticas propias hacia la actividad turística a desarrollarse y, por último, se nota organización comunitaria para la ejecución de dicha actividad.

Una vez analizados los macroelementos e identificadas las falencias en cuanto a la calidad del servicio, se aplica la gráfica denominada Torres del Servicio (instrumento de valoración) con el fin de determinar el distanciamiento o proximidad entre el beneficio esperado del cliente respecto al beneficio obtenido; posteriormente, en conjunto con la evaluación de los principales momentos de verdad obtenidos del triángulo del servicio, se lleva a cabo un análisis FODA a fin de determinar las principales fortalezas y debilidades; para finalizar, se establecen las estrategias necesarias de competitividad para potenciar el producto turístico.

\section{RESULTADOS}

A continuación, se presentan los resultados obtenidos de la metodología propuesta por The Nature Conservancy.

Tabla 1 Resultados cualitativos parámetros TNC

Parámetros

Recurso turístico

\section{Comunidad de Zuleta}

La comunidad de Zuleta tiene varios atractivos turísticos, de los que se destacan, en cuanto a atractivos naturales, el bosque protector y la 
laguna Zuleta. En cuanto a atractivos culturales, la comunidad es reconocida por los bordados, alfarería y artículos de madera ${ }^{1}$.

\section{Accesibilidad}

\section{Protección atracciones en cuanto posibles impactos}

Seguridad

\author{
Autoridad y \\ manejo \\ administrativo
}

Financiamiento

\section{Compromiso de} actores

\section{Aporte a la comunidad local Infraestructura y facilidades}

El acceso a la comunidad cuenta con vías de primer orden, como la vía Colectora Quito-Cayambe y el nuevo paso lateral Zuleta. La distancia desde Quito es de $61.6 \mathrm{~km}$ aproximadamente $2 \mathrm{~h} 00$ y $15.9 \mathrm{~km}$ partiendo de lbarra con un tiempo aproximado de 30 minutos.

Según el informe técnico del MAE, en el año 2006 se reconoce que dentro de la parroquia existen 4 zonas potenciales con restauración pasiva (protección de vegetación aledaña a lagos); 4 zonas de restauración activa para contrarrestar procesos erosivos; y 14 áreas para protección de recursos hídricos. En esas zonas se establecen actividades que permiten el cuidado y mitigación de impactos con respecto a varias actividades incluyendo el turismo (Gobierno Autónomo Desentralizado de la Parroquia Angochagua, 2015).

Según el PDOT de Angochagua, la comunidad de Zuleta es segura ya que cuenta con una unidad de policía comunitaria y brigadas de seguridad financiadas con presupuestos participativos (Gobierno Autónomo Desentralizado de la Parroquia Angochagua, 2015).

Su máxima autoridad es el síndico de la comunidad quien mantiene el manejo administrativo con efectividad.

Zuleta posee recursos públicos y actividades económicas como la agricultura que es el mayor ingreso de la comunidad con un $20.5 \%$, las artesanías representan el segundo rubro con un $17.11 \%$. Estas actividades contribuyen a potencializar el turismo en la zona ya que el mismo representa apenas el $1.6 \%$ según el informe realizado en el PDOT de la parroquia de Angochagua (Gobierno Autónomo Desentralizado de la Parroquia Angochagua, 2015).

Los actores públicos y privados mantienen constantes lazos a fin contribuir al proyecto.

La aplicación de proyectos turísticos en la comunidad pretende la contribución directa con ella.

Según la observación realizada, la comunidad como tal cuenta con hospedaje comunitario y un modelo de cabaña de lujo para acoger a los turistas, además de un comedor comunitario extenso. Por otro lado, según el catastro del 2016, la parroquia de Angochagua no registra establecimientos; sin embargo, existe información de San Antonio con tres establecimientos, un restaurante y dos cafeterías registradas.

\footnotetext{
${ }^{1}$ De acuerdo a la jerarquización realizada por la Universidad Central del Ecuador y en concordancia con la información proporcionada por el GAD de Angochagua, se establece cuatro atractivos de importancia en la localidad Zuleta. Bosque Protector "Zuleta y Anexos Cía. Ltda." Y laguna Zuleta con jerarquía II debido a que son importantes para el mercado interno debido a que los servicios de apoyo son mínimos (accesibilidad, infraestructura). Por otro lado, las artesanías (bordados, alfarería, artículos en madera) son consideradas de jerarquía III debido a que representan el rasgo llamativo para el mercado interno o externo.
} 
Diana Enríquez - Paola Flores - Andrea Vásquez

Estudio preliminar de sitio y competitividad en propuestas turísticas comunitarias:

Zuleta paisaje cultural

Salinas registra dos restaurantes y una cafetería, y Ambuquí cuenta con cuatro restaurantes y una fuente de soda, entre otros (Ministerio de turismo, 2016).

Alcance y mercado potencial
La provincia de Imbabura representa el $13.5 \%$ del turismo nacional destacando sobre todo los cantones de Otavalo y Cotacachi, los cuales han sido principalmente puntos receptivos para el turismo extranjero. La demanda de turistas nacionales que llegan a la provincia en un $43 \%$ proviene de la ciudad de Quito, esto debido a la cercanía que existe, mientras que la demanda extranjera con un $41 \%$ proviene principalmente de Colombia debido a la cercanía geográfica y al comercio existente entre ambos países. Zuleta no cuenta con un registro específico de visitantes; sin embargo, de acuerdo a los registros oficiales de lbarra, se ha obtenido que de los turistas nacionales y extranjeros que arriban a esta ciudad, el 4\% llega a Zuleta.

Fuente: Entrevista a comuneros y registro de ficha técnica PDOT. Angochagua. Ibarra Turística en cifras.

La primera evidencia de los resultados obtenidos reporta cualitativamente el análisis de diez puntos estratégicos básicos para considerar a un lugar adecuado para ejercer la actividad turística, los cuales resultan favorables para la inclusión de esta actividad en la comunidad.

Tabla 2 Parámetros cuantitativos parámetros TNC

\begin{tabular}{|c|c|c|c|c|}
\hline & Factores de evaluación preliminar & Cumple & $\begin{array}{c}\text { Cumple } \\
\text { parcialmente }\end{array}$ & No cumple \\
\hline 1 & $\begin{array}{l}\text { ¿Existe alguna atracción natural } 0 \\
\text { cultural? }\end{array}$ & 3 & & \\
\hline 2 & $\begin{array}{l}\text { ¿Se pueden establecer fácilmente } \\
\text { accesos? }\end{array}$ & 3 & & \\
\hline 3 & $\begin{array}{l}\text { ¿Se pueden proteger las atracciones de } \\
\text { impactos causadas por visitantes a un } \\
\text { nivel aceptable? }\end{array}$ & 3 & & \\
\hline 4 & $\begin{array}{l}\text { ¿El área está libre de problemas de } \\
\text { seguridad? }\end{array}$ & & 2 & \\
\hline 5 & $\begin{array}{l}\text { ¿El área tiene suficiente autoridad de } \\
\text { manejo y administración? }\end{array}$ & 3 & & \\
\hline 6 & $\begin{array}{l}\text { ¿Existen expectativas razonables de } \\
\text { financiamiento? }\end{array}$ & 3 & & \\
\hline 7 & $\begin{array}{l}\text { ¿Los directores, operadores de turismo y } \\
\text { comunidad desean adjudicarse a los } \\
\text { lineamientos de turismo responsable? }\end{array}$ & 3 & & \\
\hline 8 & $\begin{array}{l}\text { ¿Las visitas mejoran la salud de la } \\
\text { biodiversidad, apoyo a la cultura o reduce } \\
\text { la pobreza en los grupos sociales? }\end{array}$ & 3 & & \\
\hline 9 & ¿Existen facilidades turísticas? & & 2 & \\
\hline
\end{tabular}


Diana Enríquez - Paola Flores - Andrea Vásquez

Estudio preliminar de sitio y competitividad en propuestas turísticas comunitarias:

Zuleta paisaje cultural

10 ¿Se puede identificar de forma preliminar 3

el mercado objetivo potencial?

TOTAL

24

4

0

Fuente: Observación directa y registro de ficha técnica

Tabla 3 Parámetros de evaluación

\section{Parámetros de evaluación}

El producto turístico cumple

El producto turístico cumple parcialmente

El producto turístico no cumple

Las cifras evidenciadas en los resultados de la Tabla $N .{ }^{\circ} 2$ reportan que el producto turístico cumple con la mayoría de factores de evaluación preliminar en un $85,71 \%$ y, parcialmente, el producto cumple con un $14,29 \%$, datos que resultan favorables para la categorización de dicho producto turístico. Es importante mencionar que las cifras de valoración, así como la definición para la caracterización del producto, están basadas en el documento técnico de planificación para la conservación de sitios sustentada por la metodología Nature Conservancy el mismo que cuenta con parámetros pre establecidos.

Tabla 4 Categorización del producto turístico

\begin{tabular}{lll}
\hline \multicolumn{1}{c}{ Resultados } & \multicolumn{1}{c}{ Valoración } & \multicolumn{1}{c}{ Observaciones } \\
\hline Producto estrella & 26 a 30 puntos & $\begin{array}{l}\text { El producto está listo para iniciar } \\
\text { operación turística. }\end{array}$ \\
El producto presenta ciertas \\
Producto A
\end{tabular}

De acuerdo con los resultados obtenidos en la Tabla N ․ํ 2 , el producto tiene una valoración de 24 puntos con posibilidades de ejercer la actividad turística después de solucionar las falencias identificadas.

Tabla 5 Análisis de los elementos básicos que componen el producto turístico Zuleta.

\section{Elementos a considerarse}

\section{Descripción}

La comuna Zuleta es una de las comunidades del norte del país más reconocidas. En la zona de Ibarra, la hacienda Zuleta es un producto 


\section{Accesibilidad}

\section{Planta Turística}

\begin{abstract}
consolidado para el mercado internacional; la comunidad permanece aislada de esta iniciativa privada y pretende consolidar un producto turístico que beneficie al grupo social. Se pretende brindar una experiencia diferente con atractivos naturales y culturales.
\end{abstract}

Las vías que permiten el acceso a la comunidad son de primer orden, existe transporte público y privado, la ubicación es adecuada cerca de la ciudad de Ibarra a una distancia prudente de Quito y el aeropuerto.

En la comunidad existe una hospedería comunitaria "Hospedaje Carmita", en la que los turistas conviven con una familia, además cuenta con una propuesta piloto de cabaña para hospedaje de turistas con todas las facilidades enfocadas para personas con un alto poder adquisitivo. Por otro lado la comunidad también cuenta con varios lugares de comida propios de señoras de la comunidad y un comedor comunitario.

\title{
Fuente: Observación directa- Ficha técnica
}

Los resultados evidenciados en la Tabla $N . \circ 5$ nos muestran la realidad actual que tiene la comunidad basado en los elementos que deben componer un producto de este tipo, los cuales indican avances favorables para la aplicación de esta actividad y el desarrollo productivo.

En cuanto a la valoración de la calidad de los servicios prestados actualmente en la comunidad, se presentan los resultados obtenidos aplicando la segunda metodología.

Tabla 6 Percepción del servicio recibido

$\begin{array}{cc}\text { NN. } .^{-} \quad \text { Componentes del destino } & \begin{array}{c}\text { \% calificación por } \\ \text { elemento }\end{array}\end{array}$

\begin{tabular}{lll}
\hline $\mathbf{1}$ & Vías de acceso & $80 \%$ \\
$\mathbf{2}$ & Acceso a servicios básicos & $90 \%$ \\
$\mathbf{3}$ & Alojamiento y alimentación & $50 \%$ \\
$\mathbf{4}$ & Senderos de interpretación en el bosque & $30 \%$ \\
$\mathbf{5}$ & Calidad en las artesanías y bordados & $90 \%$ \\
$\mathbf{6}$ & Actividades preparadas para el visitante & $50 \%$ \\
$\mathbf{7}$ & Predisposición de la comunidad & $50 \%$ \\
$\mathbf{8}$ & Sanitación y sanidad & $50 \%$ \\
$\mathbf{9}$ & Políticas administrativas para turismo & $75 \%$ \\
\hline
\end{tabular}


10 Organización comunitaria para el desarrollo de turismo $60 \%$

Fuente: Encuestas visitantes.

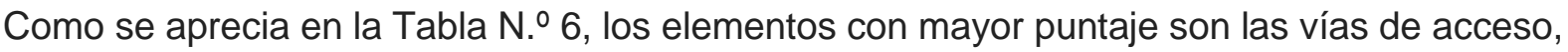
servicios básicos y calidad de las artesanías y bordados, el elemento de menor puntaje es senderos de interpretación en el bosque. Cifras que evidencian un desequilibrio en la percepción obtenida y muestran indicios de las principales falencias que tiene el producto.

Beneficio esperado

Porcentajes

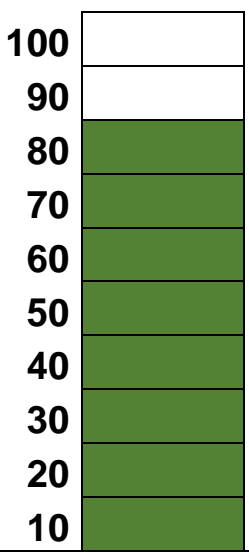

Beneficio percibido

Porcentajes

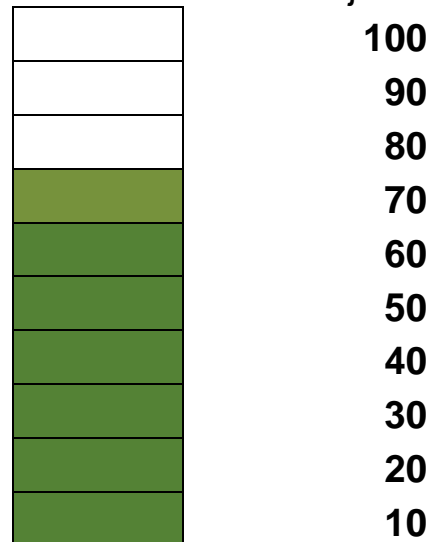

Gráfico 3: Torres del servicio (Evaluación del cliente externo). Fuente: Encuesta visitantes.

En el gráfico, se muestra que los visitantes tienen una expectativa alta con respecto a los servicios ofrecidos en la comunidad; sin embargo, la percepción de la experiencia fue inferior a sus expectativas, lo que da como resultado visitantes insatisfechos.

\section{Causas de la asimetría entre las dos torres}

Según los comentarios emitidos por los visitantes, las causas de la asimetría entre las torres son las siguientes:

1. Se espera realizar actividades junto con los comuneros, no solo observar las actividades que realizan.

2. Se esperaba mayor calidad en los productos de alojamiento y alimentación (oferta limitada).

3. La guianza en el bosque no es adecuada ya que se carece de información turística.

Por lo tanto, estas serían las actividades que deben mejorarse o potencializarse en la oferta de turismo comunitario de Zuleta, para lo cual se procede a analizar los procesos de momentos de verdad a través del esquema FODA.

Tabla 7 Momentos de verdad: Debilidad/Fortaleza. 
Estudio preliminar de sitio y competitividad en propuestas turísticas comunitarias:

Zuleta paisaje cultural

Registro en hospedería

Visita al proyecto de bordadoras

Caminata en el Bosque

Servicio de alimentación

Venta de artesanías

\begin{tabular}{ll}
$X$ & $x$ \\
$X$ & $x$ \\
\hline
\end{tabular}

Con la información obtenida en la Tabla $N$ ․ㅜ 6 , se escoge como fortaleza la visita al proyecto de bordadoras ya que registra alta aceptación y como debilidad se establecen las caminatas en el bosque de Zuleta debido a que no tiene un buen porcentaje de aceptación y es una de las causas de asimetría en la torre de servicio.

Tabla 8: Factores que influencian la fortaleza

Momento de la verdad:

visita al proyecto

Factores de influencia

bordadoras

$\begin{array}{ll}\mathbf{1} & \text { Atractivo conocido } \\ \mathbf{2} & \text { Productos de calidad } \\ \mathbf{3} & \text { Organización de las trabajadoras } \\ \mathbf{4} & \text { Espacio adecuado de exposición y venta } \\ \mathbf{5} & \text { Las señoras comentan del proceso a los clientes en forma } \\ \mathbf{6} & \text { adecuada } \\ & \text { El servicio turístico es de calidad y calidez }\end{array}$

Tabla 9 Factores que influencian la debilidad

Momento de la verdad: caminata en el bosque

Factores de influencia

protector

$\begin{array}{ll}\mathbf{1} & \text { Falta de señalética adecuada } \\ \mathbf{2} & \text { Senderos poco definidos } \\ \mathbf{4} & \text { Personal poco capacitado para la guianza } \\ & \text { Migración de los comuneros a las grandes ciudades, que deja } \\ & \text { como consecuencia una población longeva en su mayoría, } \\ & \text { perdiendo pertenencia e identidad y reduciendo } \\ & \text { considerablemente la población económicamente activa. } \\ & \text { Espacio demasiado amplio y poca delimitación de los senderos } \\ & \text { atractivos }\end{array}$

En función del análisis realizado se determinan áreas defensivas de iniciativa estratégica en las que se valoran del 1 al 5 , considerando 1 como el puntaje más bajo en función de la variable analizada y 5 el puntaje más alto. Con dos principales variables:

1. Promoción de las manifestaciones culturales de la comunidad, en específico los bordados.

2. Fortalecimiento del patrimonio natural a través de la manifestación cultural del uso de plantas medicinales encontradas en el páramo. 
Diana Enríquez - Paola Flores - Andrea Vásquez

Estudio preliminar de sitio y competitividad en propuestas turísticas comunitarias:

Zuleta paisaje cultural



Tabla 10 Fortalezas y oportunidades

\section{Estrategias}

- EF: Consolidación de la marca de manufactura de los bordados de Zuleta a través de organismos de apoyo (Estrategia de diferenciación de producto de acuerdo a las características del servicio, (Porter 1982)).

- EO: Financiamiento del proyecto de bordados de Zuleta a través de fuentes gubernamentales como Corporación Financiera Nacional (CFN) (Estrategia de diferenciación de producto de acuerdo a las características de la empresa, (Porter 1982)). 
Diana Enríquez - Paola Flores - Andrea Vásquez

Estudio preliminar de sitio y competitividad en propuestas turísticas comunitarias: Zuleta paisaje cultural

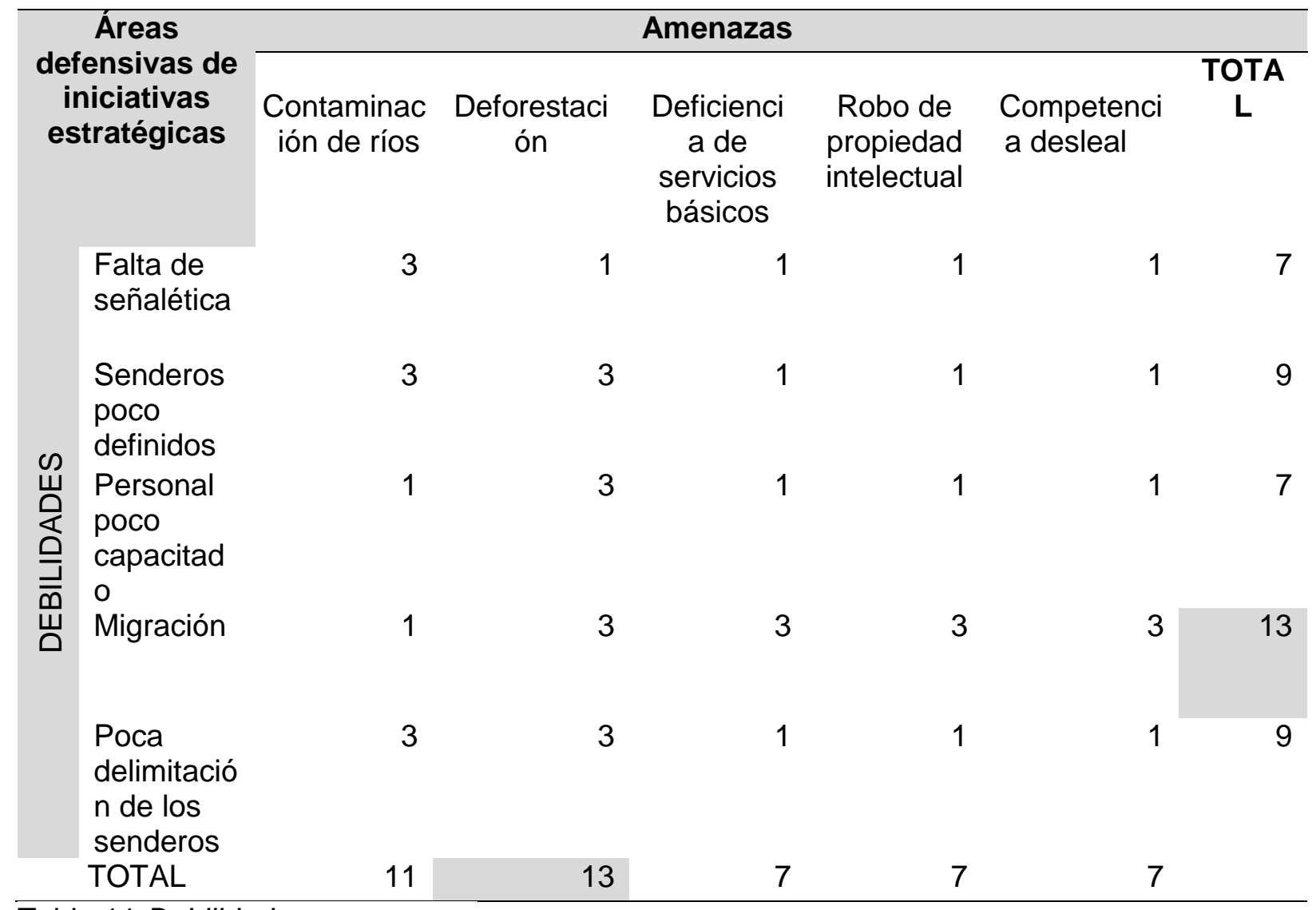

Tabla 11 Debilidades y amenazas

\section{Estrategias}

- EA: Búsqueda de apoyo de instituciones del Estado como el Ministerio de Turismo y de Medio Ambiente para la protección de estas áreas Estrategia de diferenciación de producto de acuerdo a las características de la empresa, (Porter 1982).

- ED: Programa de capacitación en servicio turístico para la población económicamente activa a fin de evitar la migración Estrategia de diferenciación de producto de acuerdo a las características del servicio, (Porter 1982).

\section{DISCUSIÓN}

En el presente estudio se confirma que la comuna Zuleta es dueña de una diversidad de elementos naturales y manifestaciones culturales, lo que la convierte en un destino turístico atractivo que, a su vez, abre la posibilidad de nuevos estudios en cuanto al patrimonio natural y cultural de la localidad.

De acuerdo al Plan Nacional del Buen Vivir (2013), es necesario fortalecer mecanismos para garantizar la conservación de la propiedad de las tierras comunitarias y la posesión de los territorios ancentrales de las comunidadespara evitar su desplazamiento. Se hace necesario desarrollar alternativas económicas, sociales y ecológicas que permitan la conservación de estos territorios y mejoren las condiciones de vida de los comuneros para evitar la migración hacia las ciudades más cercanas y mantener intacto el patrimonio tangible e intangible.

Es necesario aplicar un plan de acción que defina el paisaje de conservación tanto para especies como comunidades, debido a que cada grupo social, en su práctica cotidiana, es portador de una cultura propia que se manifiesta en sus creencias, vivencias y tradiciones. 


\section{Estudio preliminar de sitio y competitividad en propuestas turísticas comunitarias: Zuleta paisaje cultural}

El desarrollo de un programa que fomente en los grupos sociales la necesidad de la conservación y manejo de sus recursos para el bien común es necesario; sin embargo, es importante realizar estudios que pemitan determinar si existe o no el potencial para generar un mayor desempeño en el ámbito turístico, ya que no necesariamente esta actividad es adecuada para todos los territorios. Lo más grave es que, de no hacer los estudios pertinentes, se corre el riesgo de que las comunidades dejen de realizar sus actividades propias como la ganadería, agricultura y, posiblemente, mermen su desarrollo.

Los resultados obtenidos de The Nature Conservancy (1999), con los que se da cumplimiento al objetivo general de este trabajo, han revelado que Zuleta cumple en un $85,71 \%$ con los parámetros básicos para considerarla como un lugar adecuado para ejercer la actividad turística, además de haber obtenido una valoración de 24 puntos sobre 30.

Cabe mencionar que, una vez aplicada la metodología, se puede recomendar para próximas investigaciones variar los parámetros TNC con elementos más apegados a las necesidad de la comunidad y su participación en el desarrollo de la actividad turística. Por otro lado, es necesario en la medición cuantitativa utilizar herramientas o datos valederos 0 verificables a fin de no caer en la apreciación básica del investigador y por tanto volver la investigacion subjetiva.

Por otro lado, los resultados obtenidos de la metodología propuesta por Albrecht y Zemke (1988) han revelado que existe un incremento en la percepción del cliente en cuanto a los elementos esenciales de cobertura de servicios básicos para los turistas y la calidad en las artesanías y bordados de la comunidad con un $80 \%$ y $90 \%$ respectivamente. Sin embargo, se evidencia una notable disminución de esta percepción en los procesos esenciales del destino turístico como senderos de interpretación en el bosque protector y predisposición de la comunidad para atender al turista con un 30\% y 50\% respectivamente; estos factores han sido identificados como las falencias iniciales de la calidad del servicio. Por otro lado, al determinar el distanciamiento o proximidad entre el beneficio esperado del cliente, con respecto al beneficio obtenido, los resultados manifiestan que los clientes tienen una expectativa alta con respecto a los servicios ofrecidos en la comunidad; sin embargo, la percepción de la experiencia fue inferior a sus expectativas, lo que da como resultado clientes insatisfechos y disminuye la competitividad del producto.

Al analizar los momentos de verdad, se presentan los resultados como fortalezas, oportunidades, debilidades y amenazas que se han identificado en el producto turístico. La fortaleza esencial detectada son los bordados y, como principal oportunidad, el creciente apoyo a las comunidades. Por otro lado, entre las debilidades más relevantes se encuentra la poca predisposición de la comunidad para la prestación del servicio y la limitada adecuación técnica de los atractivos e instalaciones para lo cual se deben aplicar estrategias de diferenciación.

En cuanto a la metodología aplicada, es importante determinar que la medición de los momentos de verdad con los visitantes brinda datos referenciales y son la base para ejecutar estrategias de calidad, estos datos son subjetivos y pueden estar influenciados por condiciones que no pueden ser manejados (mal clima, accidentes, disposiciones gubernamentales, entre otros) ya que el cliente valora su experiencia sin discriminar entre la oferta de servicios y otras condiciones. Por lo tanto, para mejorar la medición y aportar de mejor manera a definir estrategias de competitividad, se recomienda considerar en el FODA los criterios más relevantes a fin de establecer estrategias que permitan mejorar la calidad del servicio del producto turistico, y afianzar la competitividad de la propuesta. Por otro lado, se hace necesario, en próximas investigaciones, validar que tan aptas son las estrategias propuestas y elaborar un análisis ampliado de las mismas, es decir esta información puede aportar a la generación de un plan estratégico de desarrollo para la localidad.

Así mismo, se especifica que, aunque los resultados obtenidos en este artículo responden favorablemente a la aplicación de un modelo de desarrollo intregral con bases productivas en el turismo, es necesario realizar nuevos estudios que permitan identificar otras actividades pertinentes con el grupo social; también se plantea la existencia de un importante reto que es crear sensibilidad, conciencia, entendimiento y compromiso en la población a fin deconservar 


\section{Diana Enríquez - Paola Flores - Andrea Vásquez \\ Estudio preliminar de sitio y competitividad en propuestas turísticas comunitarias: \\ Zuleta paisaje cultural}

el patrimonio histórico, cultural y ambiental de un pueblo, generando pertinencia en su identidad y legado a fin de consolidar un producto turístico de calidad que sea sostenible y diferenciado.

\section{BIBLIOGRAFÍA}

Albrecht, K. y Zemke, R. (1988). Gerencia a través del Triángulo del servicio. En Gerencia del Servicio. Bogotá: Legis Editores.

Alprecht, K. (1992). Servicio al cliente interno. Boletín de lectura sociales y económicas, 14-23.

Alvear, G., Quishpe, C. y Enríquez , D. (2016). Turismo comunitario como alternativa de desarrollo en la comunidad de. Revista Publicando, 203-219.

Amaya, M. y Magaña, P. (2017). Evaluación de destinos turísticos mediante la tecnología de la ciencia de datos. Estudios y perspectivas en turismo , 286-305.

Bolagay, C. y Velásquez, E. (julio de 2014). Repositorio Digital. Obtenido de Universidad Técnica del Norte:

http://repositorio.utn.edu.ec/bitstream/123456789/4106/1/05\%20FECYT\%202030\%20TESIS .pdf

Carlzon, J. (1987). Moments of truth. Ballinger: Cambridge.

Colina, J. (Febrero de 2015). http://www.ucipfg.com/. Obtenido de /Repositorio/MGTS/MGTS14/MGTSV-09/semana2/Mercadeo_Turisticwww.ucipfg.como__LS2.2._Concepto_de_Producto_Turistico.pdf

García, L. (2014). Gestión. Obtenido de http://gestion.pe/atuservicio/2014/03/el-triangulo-delservicio.html

Gobierno Autónomo Descentralizado de San Miguel de Ibarra . (2012). Gobierno Autónomo Descentralizado de San Miguel de Ibarra . Obtenido de https://www.touribarra.gob.ec/esp/index.php/parroquia-de-angochagua

Gobierno Autónomo Descentralizado de San Miguel de Ibarra. (2012). http://app.sni.gob.ec/. Obtenido de http://app.sni.gob.ec/snilink/sni/PDOT/ZONA1/NIVEL_DEL_PDOT_CANTONAL/IMBABURA/IBARRA/INFORMACION_G $\mathrm{AD} /$

Iglesias, A. (s.f.). IDE-CESEM Business School Madrid. Recuperado el 18 de Julio de 2017, de http://www.formacionparaprofesionales.es/servicio-cliente-perspectiva-logistica/

INEC. (2010). http://www.ecuadorencifras.gob.ec/. Obtenido de http://www.ecuadorencifras.gob.ec//wp-content/descargas/Manu-lateral/Resultadosprovinciales/imbabura.pdf

INEC. (2010). Información Censal Cantonal . Obtenido de http://www.inec.gob.ec/cpv/index.php?option=com_contentyview=articleyid=232yltemid=1 28ylang=es

INEC. (2010). Resultados del Censo 2010 de problación y vivienda en el Ecuador. Obtenido de Fascículo Provincial Imbabura: http://www.ecuadorencifras.gob.ec/wpcontent/descargas/Manu-lateral/Resultados-provinciales/imbabura.pdf 


\section{Diana Enríquez - Paola Flores - Andrea Vásquez \\ Estudio preliminar de sitio y competitividad en propuestas turísticas comunitarias: Zuleta paisaje cultural}

Kiesecker, J., Comendant, T., Grandmason, T., y Elizabeth, G. (2007). Conservation easements in context: a quantitative analysis of their use by The Nature Conservancy. Frontiers in Ecology and the Environment.

Koontz, H. (1994). Administración una perspectiva global (Désima ed.). Mexico: Mc Graw Hill.

Koontz, H. (1999). Administración una perspectiva global (Oncena ed.).

Lincango, J. (2013). Grupos étnicos del Ecuador. Obtenido de Andres Info: http://www.andes.info.ec/es/noticias/zuleta-pequena-localidad-ecuador-preservatradicion-bordado-mano.html

Manzato, F. y Rejowski, M. (2007). Turismo cultural: Evaluación del potencial turístico de sitios arqueológicos. Estudios y perspectivas en turismo , 72-91. Obtenido de http://www.redalyc.org/articulo.oa?id=180713890004

March, I., Cabral, H. y Echeverría, Y. (2010). Programa de adaptación al cambio climático. Obtenido de The Nature Conservancy: http://www.cakex.org/sites/default/files/project/documents/TNC\%20Project\%20Summary. pdf

Ministerio de turismo. (2016). Catastro . Quito.

Ministerio de Turismo del Ecuador. (s.f.). Ecuador, potencia turistica. Recuperado el 18 de Julio de 2017, de http://servicios.turismo.gob.ec/index.php/portfolio/turismo-cifras/20-ofertaturistica/servicios-turisticos/247

Moore, A., Drumm, A., Soles, A., Patterson, C., y Terborgh, J. (2004). Desarrollo y Manejo del Ecoturismo: un Manual para los Profesionales de la Conservación.

Pereiro, X. (2013). Los efectos del turismo en las culturas indígenas. Revista Española de Antropología Americana, 155-174.

Plan Nacional del Buen Vivir. (2013). Objetivo 2 2013-2017. Obtenido de Auspiciar la igualdad, cohesión, inclusión y equidad social y territorial en la diversidad: http://www.buenvivir.gob.ec/descarga-objetivo

Prieto, J. (2014). Gerencia del Servicio. La clave para ganar todos. Bogotá: Eco Ediciones.

Requena, M., y Serrano, G. (2007). Repositorio Universidad Católica Andrés Bello. Obtenido de Biblioteca Virtual:

http://biblioteca2.ucab.edu.ve/anexos/biblioteca/marc/texto/AAR1459.pdf

Siebold, M. (18 de Julio de 2017). Mercadeo. Obtenido de http://www.mercadeo.com/blog/1967/09/momentos-de-verdad-en-la-administracion-delservicio/

Silva, A., Aguilar, Z., y HIdalgo, P. (2009). Zuleta, un nuevo paraje de alpacas. Lecciones aprendidas en el Proyecto de Manejo y Aprovechamiento Sustentable de Alpacas en los Páramos de Zuleta. Quito: EcoCiencia-PPA. Obtenido de http://ecociencia.org/zuleta-nuevo-paraje-de-alpacaslecciones-aprendidas-del-proyecto-de-manejo-y-aprovechamiento-sustentable-de-alpacasen-los-paramos-de-zuleta/

Sostenibilidad del Patrimonio . (2017). unesco.org . Obtenido de http://www.es.unesco.org 
Diana Enríquez - Paola Flores - Andrea Vásquez

Estudio preliminar de sitio y competitividad en propuestas turísticas comunitarias: Zuleta paisaje cultural

The Nature Conservancy. (1999). Planificación para la conservación de sitios. Obtenido de file://C:/Users/USER4/Downloads/metodolog\%C3\%ADa\%20Nature\%20Conservancy.pdf

UNESCO. (2005). WHC. Obtenido de Directrices Prácticas para la aplicación de la Convención del Patrimonio Mundial: http://whc.unesco.org/archive/opguide05-es.pdf

UNESCO. (s.f.). Cultural Landscapes. Obtenido de http://whc.unesco.org/en/culturallandscape/ 
FICHA TÉCNICA DE EVALUACIÓN PRELIMINAR DE SITIO

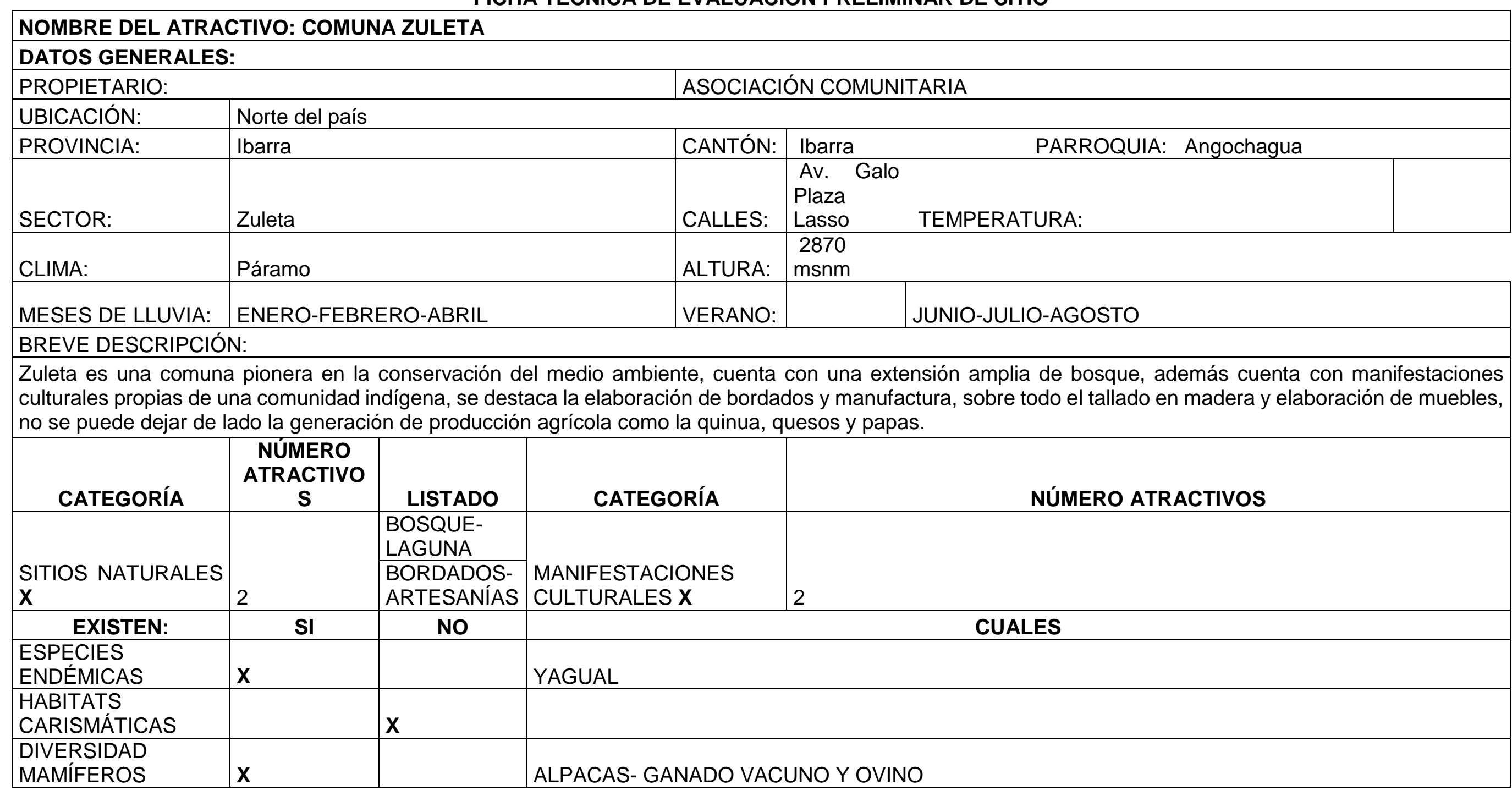


Diana Enríquez - Paola Flores - Andrea Vásquez

Estudio preliminar de sitio y competitividad en propuestas turísticas comunitarias: Zuleta paisaje cultural

\begin{tabular}{|c|c|c|c|c|c|c|c|c|c|}
\hline $\begin{array}{l}\text { FORMACIONES } \\
\text { GEOMORFOLÓGICA } \\
\text { S }\end{array}$ & & $\mathbf{X}$ & & & & & & & \\
\hline \multicolumn{2}{|l|}{ ACCESIBILIDAD } & ALTA & $\mathbf{x}$ & MEDIA & & BAJA & & & \\
\hline \multirow[b]{2}{*}{ TIPO TRANSPORTE } & \multirow[b]{2}{*}{ SUBTIPO } & \multirow{2}{*}{$\begin{array}{l}\text { ESTADO DE } \\
\text { VÍAS }\end{array}$} & & \multicolumn{4}{|c|}{ FRECUENCIA } & \multicolumn{2}{|c|}{ TEMPORALIDAD-ACCESO } \\
\hline & & & & DIARIA & SEMANAL & $\begin{array}{c}\text { MENSUA } \\
\text { L }\end{array}$ & EVENTUAL & \multicolumn{2}{|c|}{ DÍAS AL AÑO } \\
\hline \multirow{4}{*}{ TERRESTRE } & ASFALTO & $\mathrm{X}$ & BUS & $\mathrm{X}$ & & & & \multicolumn{2}{|c|}{$\begin{array}{l}\text { TODOS LOS DÍAS DEL AÑO ES } \\
\text { TRASNPORTE PÚBLICO }\end{array}$} \\
\hline & LASTRADO & & AUTOMÓVIL & $x$ & & & & & \\
\hline & EMPEDRADO & & $4 \times 4$ & & & & & \multicolumn{2}{|c|}{ TEMPORADA-VISITA } \\
\hline & SENDERO & & TREN & & & & & \multirow{2}{*}{ CULTURALES: } & JUNIO \\
\hline \multirow{3}{*}{ ACUÁTICO } & MARÍTIMO & & BARCO & & & & & & \\
\hline & FI IVIAI & & BOTE & & & & & \multirow{2}{*}{ NATURALES: } & JULIO \\
\hline & TLUVIAL & & CANOA & & & & & & AGOSTO \\
\hline \multirow{3}{*}{ AÉREO } & & & AVION & & & & & \multicolumn{2}{|l|}{ HORAS- DIA } \\
\hline & & & AVIONETA & & & & & CULTURALES & $10: 30$ \\
\hline & & & & & & & & NATURALES: & 18: 00/13:00 \\
\hline DISTANCIA: & KM & TIEMPO & \multicolumn{7}{|c|}{ OBSERVACIONES: } \\
\hline $\begin{array}{l}\text { AEROPUERTO } \\
\text { CERCANO }\end{array}$ & $59 \mathrm{~km}$ & $1: 20 \mathrm{~min}$ & \multirow{3}{*}{\multicolumn{7}{|c|}{$\begin{array}{l}\text { Existen dos rutas, el nuevo desvío desde Cayambe, permite un rápido acceso a la comunidad pero } \\
\text { carece de señalización adecuada, sin embargo, es de fácil determinación. }\end{array}$}} \\
\hline $\begin{array}{l}\text { CAPITAL } \\
\text { PROVINCIAL }\end{array}$ & $5 \mathrm{~km}$ & $20 \mathrm{~min}$ & & & & & & & \\
\hline $\begin{array}{l}\text { CENTRO } \\
\text { POBLADO } \\
\text { CERCANO }\end{array}$ & $1000 \mathrm{~km}$ & $10 \mathrm{~min}$ & & & & & & & \\
\hline
\end{tabular}




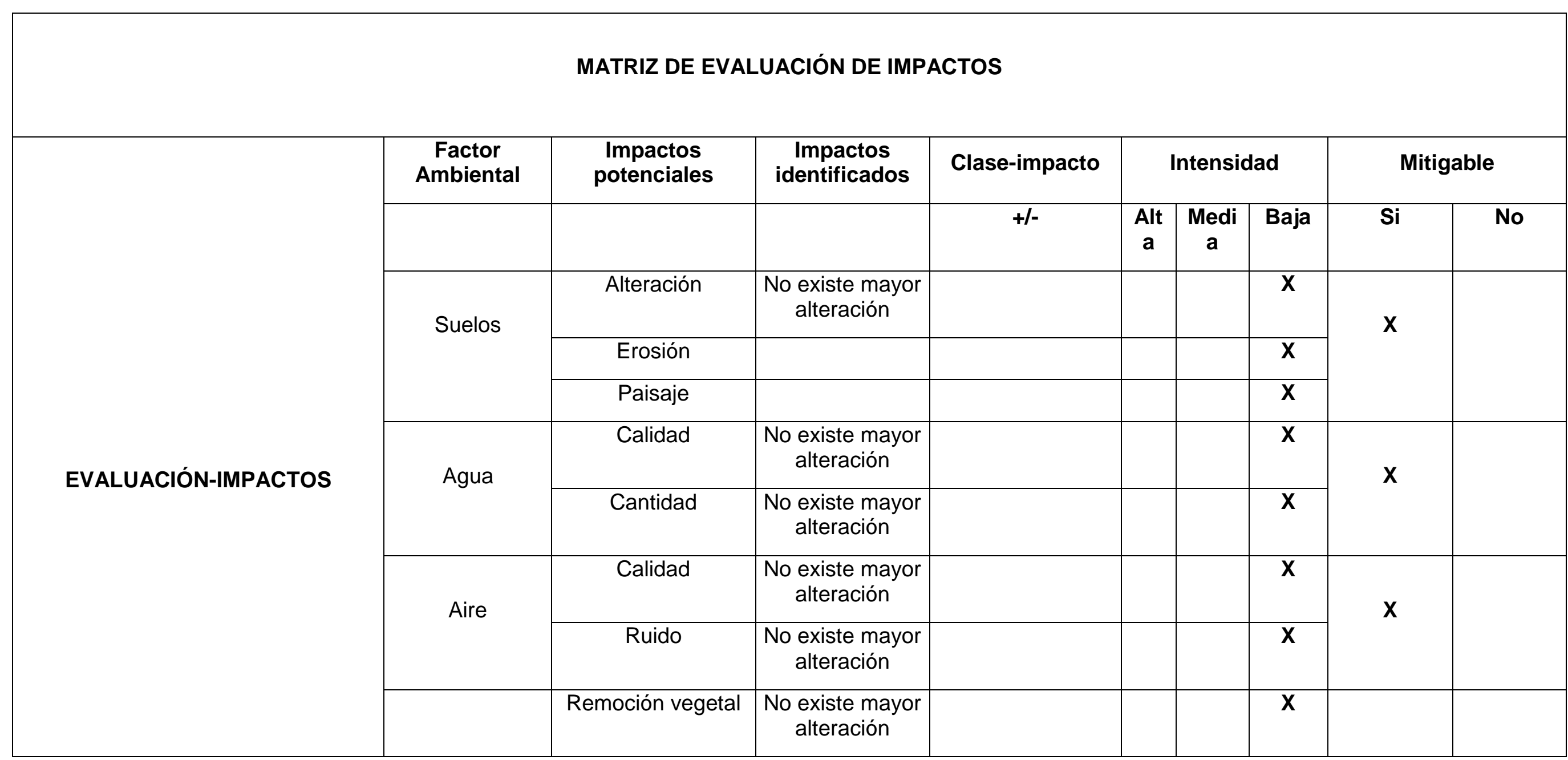


Diana Enríquez - Paola Flores - Andrea Vásquez

Estudio preliminar de sitio y competitividad en propuestas turísticas comunitarias: Zuleta paisaje cultural

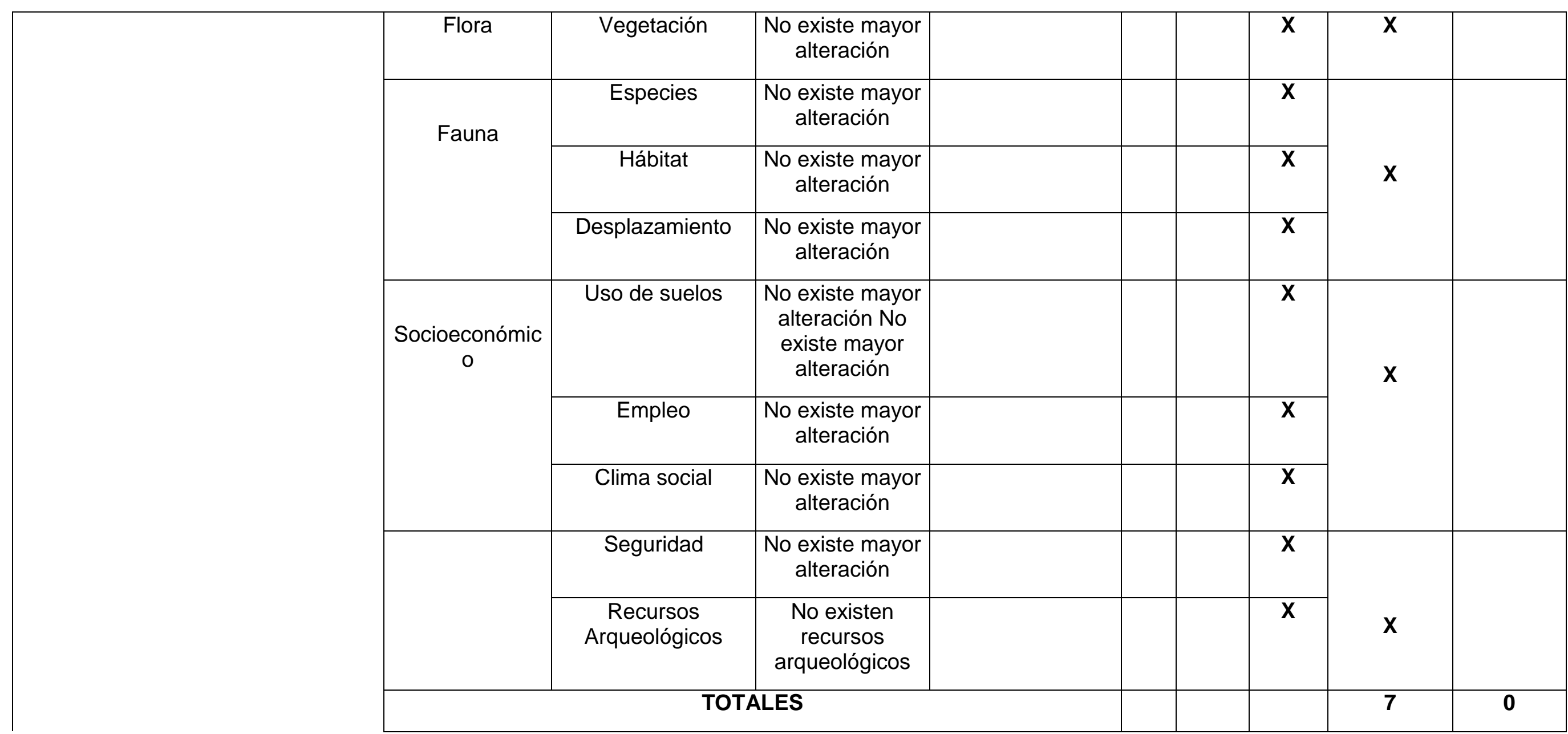




\begin{tabular}{|l|l|l|l|}
\hline & & & \\
\hline
\end{tabular}


Diana Enríquez - Paola Flores - Andrea Vásquez

Estudio preliminar de sitio y competitividad en propuestas turísticas comunitarias: Zuleta paisaje cultural

\begin{tabular}{|c|c|c|c|}
\hline \multicolumn{4}{|c|}{$\begin{array}{l}\text { ¿El área está libre de problema de seguridad que no puedan ser controlados efectivamen } \\
\text { La comunidad tiene códigos de conducta y seguridad, sin embargo existe delincuencia común. }\end{array}$} \\
\hline & SI & NO & OBSERVACIONES \\
\hline Existe un manual de seguridad para las instalaciones & & & No aplica \\
\hline $\begin{array}{l}\text { Los senderos tienen medidas de seguridad que faciliten las caminatas y } \\
\text { recorrido de los visitantes. }\end{array}$ & & $\mathbf{X}$ & $\begin{array}{l}\text { Los senderos son delimitados como una comunidad rural, } \\
\text { no existen adecuaciones turísticas. }\end{array}$ \\
\hline Existe vigilancia en la higiene de las instalaciones sanitaras & & $\mathbf{X}$ & $\begin{array}{l}\text { Las instalaciones públicas sanitarias no cumplen con } \\
\text { condiciones básicas, las hospederías y restaurante } \\
\text { comunitario si cuenta con baterías sanitarias adecuadas. }\end{array}$ \\
\hline La calidad del agua es apta para los tipos de consumo humano & $\mathbf{X}$ & & \\
\hline Los trabajadores poseen seguro & & & No aplica \\
\hline Poseen servicio de primeros auxilios & & $\mathbf{X}$ & \\
\hline Existen servicios médicos en caso de emergencia & $\mathbf{X}$ & & Propios de una comunidad rural \\
\hline Se controla el encendido- apagado de equipos y maquinarias & & & No aplica \\
\hline $\begin{array}{l}\text { El personal cuenta con equipos de seguridad adecuados para sus } \\
\text { actividades. }\end{array}$ & & $\mathbf{X}$ & \\
\hline \multicolumn{4}{|c|}{ ¿El área tiene suficiente autoridad de manejo y administración para la implementación y monitoreo de un programa de turismo responsable? } \\
\hline & SI & NO & OBSERVACIONES \\
\hline Posee un manual de funciones & & & No aplica \\
\hline Posee un organigrama estructural & $\mathbf{X}$ & & Como comunidad \\
\hline
\end{tabular}


Diana Enríquez - Paola Flores - Andrea Vásquez

Estudio preliminar de sitio y competitividad en propuestas turísticas comunitarias: Zuleta paisaje cultural

\begin{tabular}{|l|l|l}
\hline La empresa posee un plan claro y detallado de los objetivos y metas & $\mathbf{X}$ & Planes de desarrollo turístico de la comunidad
\end{tabular}

de la organización 
Diana Enríquez - Paola Flores - Andrea Vásquez

Estudio preliminar de sitio y competitividad en propuestas turísticas comunitarias:

Zuleta paisaje cultural

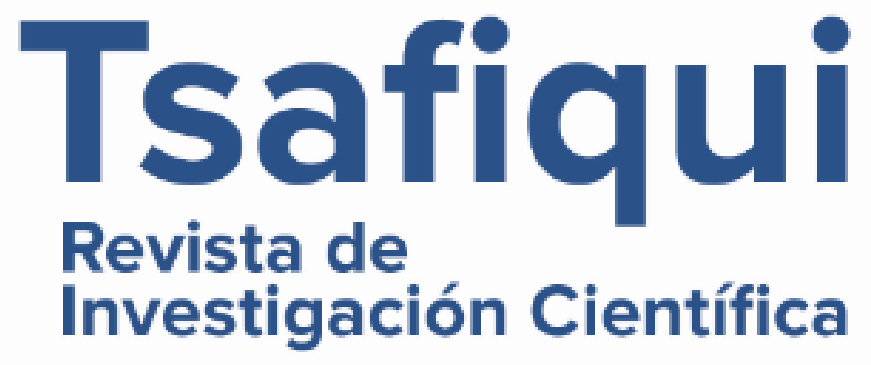

